\title{
Performance Bounds of Prioritized Access in Coexisting Cognitive Radio Networks
}

\author{
Oluwaseyi Omotere, Lijun Qian \\ Department of Electrical and Computer Engineering \\ Prairie View A\&M University, Texas A\&M University System \\ Prairie View, Texas 77446, USA \\ Email: omotere@gmail.com, liqian@pvamu.edu
}

\author{
Riku Jäntti \\ Department of Communications and Networking \\ Aalto University \\ P.O. Box 13000, 00076 Aalto, Finland \\ Email: riku.jantti@aalto.fi
}

\begin{abstract}
In this paper, we consider two uncoordinated and geographically overlapping Cognitive Radio (CR) networks coexisting together with Primary User (PU) networks. One of the CR networks, the Cognitive Gateway Network (CGN) has higher priority to access the spectrum than the other CR network. We study the achievable rates of both $C R$ networks. The achievable data rate per-node is derived analytically considering various scenarios of spectrum sensing results. The performance bound of spectrum sensing in terms of false alarm probability under prioritized access is also derived. The results of this paper illustrate how the different CR network parameters, such as transmission probability and sensing performance, affect the achievable rate in prioritized overlapping CR networks. Thus it may serve as a guide for practical deployments.
\end{abstract}

Index Terms-Cognitive radio network, prioritized secondary access, achievable rate

\section{INTRODUCTION}

High demand for wireless services has led to new spectrum sharing strategies aiming to better utilize scarce spectrum resources. Some practical scenarios include multiple Cognitive Radio (CR) networks coexisting in the same geographical area where one of the CR networks has higher priority to spectrum resources. In this paper, we consider two coexisting CR networks where the Cognitive Gateway Network $(C G N)$ has higher priority than the Cognitive Radio Network $(C R N)$. One example is in an environment where different nodes of different data requirements are deployed, assigning priority to the node ensures that such data requirements are met. Another example is in military operations, a soldier radio network and a wireless sensor network both based on cognitive radio (e.g., JTRS radios) may be deployed in the theater. The CR network of the soldiers would have a higher priority than the wireless sensor network.

The logical and physical network scenarios of two coexisting CR networks with prioritized access, $C G N$ and $C R N$, are shown in Figure 1 and Figure 2, respectively. Both CR networks are in the same spatial domain and they are using the same frequency opportunistically with a Primary User (PU). Thus, both $C G N$ and $C R N$ need to perform spectrum sensing of the PU, and $C R N$ needs to perform additional spectrum sensing of the $C G N$ since it has lower priority on spectrum access.

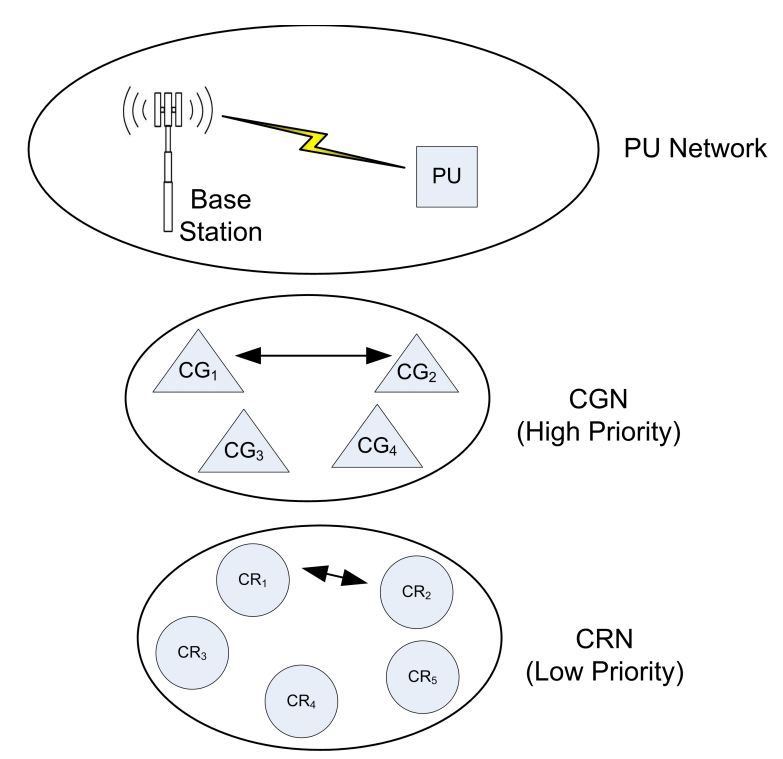

Figure 1: Logical network scenario, where two coexisting CR networks $(C G N$ and $C R N)$ have different priority of spectrum access while yielding to a primary user.

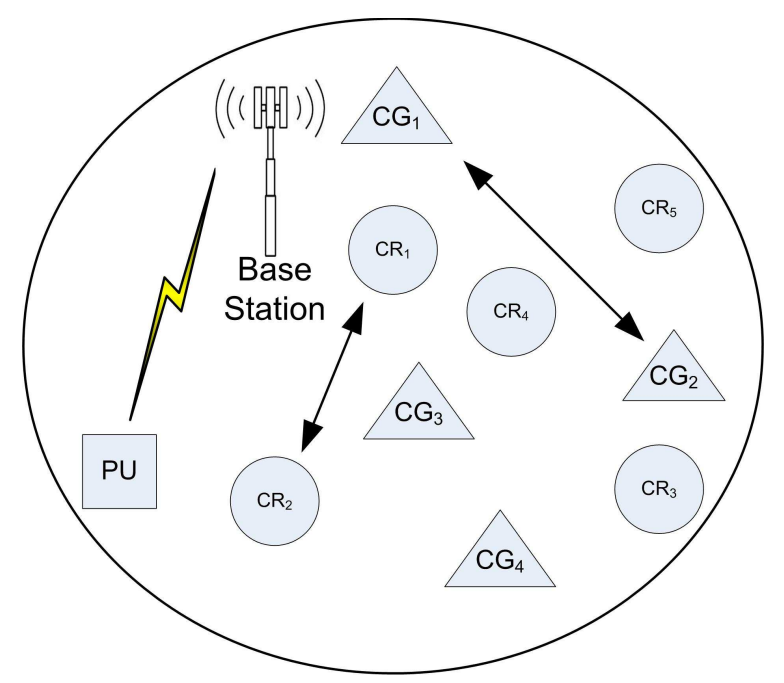

Figure 2: Physical network scenario, where two coexisting CR networks ( $C G N$ and $C R N)$ are within the range of a primary user. 
There are rich literatures on the coexistence of heterogeneous wireless networks on the ISM bands. Research work in this field has focused on the coexistence of WiFi (802.11) and Zigbee (802.15.4) radios, see, e.g., [1]-[3]. IEEE 802.11 b/g networks may interfere with IEEE 802.15.4 sensor networks and thereby introduce significant coexistence problems for low-power sensor nodes. Although intensive research has been carried out on CR technology and single CR networks, only a few studies address the coexistence of multiple CR networks [4]-[10]. However, their analysis did not consider the access priority of CR networks. For example, Nieminen et al. [4] discussed the fundamental per-node throughput of a CR user when multiple $\mathrm{CR}$ networks coexist under simultaneous access with the PU.

The main objective of this paper is to find out how much throughput a CR network can achieve in the presence of another CR network with prioritized access and a PU, and the corresponding performance bound. This bound determines whether it is feasible to deploy multiple CR networks in the same region with the required quality-of-service, say, the minimum throughput. We analyze the performance of a CR network in detail by considering the effects of various $\mathrm{CR}$ network parameters such as transmission probability, performance of spectrum sensing (false alarm probability and miss rate probability).

The paper is organized as follows. First, the system model is given in Section II. Then, we derive the per-node throughput and the performance bound in overlapping CR networks with prioritized access in Section III. Simulation results and analysis are presented in Section IV. Related works are reviewed in Section V. Section VI gives the concluding remarks.

\section{System Model}

In this paper, we focus on the case where two CR networks are uncoordinated and deployed in the same geographical area with different access priority in addition to a PU. The presence of the PU is defined using the following hypotheses. Hypothesis $H_{0}^{P U}$ denotes the case in which the PU is idle and $H_{1}^{P U}$ stands for the case in which the PU is active. We further make the following assumptions:

- The CG in $C G N$ has prioritized access to the spectrum, therefore CR in $C R N$ must sense for CG before transmitting data. $H_{0}^{C G}$ denotes the case where CG is idle and $H_{1}^{C G}$ stands for the case where CG is active.

- $P_{f, i}$ and $P_{m, i}$ are the false alarm probability and miss rate probability respectively. $P_{f, i}$ is the probability that the CR decides the PU is in operation whereas it was actually off, while $P_{m, i}$ is the probability that the CR fails to detect the presence of the PU.

- Each CR network performs its own spectrum sensing and the corresponding sensing period and probabilities of false alarm and miss rate are taken into account in this paper. However, they do not coordinate their sensing nor share the sensing results.

- Simplified frame structure with a sensing period $\tau$ and data period $T-\tau$ such that $T$ is one frame duration.
$C R N$ may need a sensing period $\tau_{2}$ longer than that of $C G N\left(\tau_{1}\right)$ to perform sensing of active CGs.

- Since CSMA/CA is a well-established Media Access Control (MAC) protocol and has been adopted by many practical wireless networks, we presume that the $\mathrm{CR}$ networks use CSMA/CA as the basis of their MAC protocol. It is also assumed that CR nodes can detect others' transmissions by using CSMA/CA, where the RTS/CTS message exchange is carried out before data transmissions.

- Furthermore on the access scheme, if CGN tries to access the medium and the medium is in use, then the CGN needs to identify whether the user is a PU or a CRN device. If the user is PU, then CGN device shall delay for some time until the channel becomes available. If the user is a CRN device, then CGN device has the right to use the channel. In this case, CRN device shall stop the use of the channel after detecting the CGN transmission. In other words, CRN ensures both the PU and CGN are not transmitting before using the channel.

- The secondary CR networks are homogeneous in the sense that the nodes in the CR networks have similar capabilities and behaviors, such as the transmission power.

- Since the CR nodes are typically less powerful than the primary nodes, have smaller transmission ranges and are located closer to each other, we model the channel between CR nodes with Rayleigh fading.

- Noise is Additive White Gaussian Noise (AWGN).

In this paper, we focus on CR ad hoc networks instead of CR networks with infrastructure support such as the IEEE 802.22 [11] systems. There is a universal detector for PU signals in each CRN while each CR node uses CSMA/CA protocol by exploiting this detection result.

\section{Per-node Throughput and Performance Bound}

Since multiple networks coexist in the same region, both intra-network and inter-network interferences will be introduced. In this section we first derive the interference model for overlapping CR networks which is then exploited to deduce the per-node throughput under prioritized access. The results were further enhanced by taking into account the sensing parameters, the probability of false alarm and miss rate.

\section{A. Interference Modelling and Probabilistic Throughput Per- node}

In this section, we derive the characteristics of interference in a Poisson Cluster Process, specifically we considered Neyman-scott Cluster Process [12]. The CRN and CGN node is modeled as Poisson Cluster Process $\phi$ on $\mathbb{R}^{2}$. The center of the clusters are assumed to be distributed according to a stationary Poisson Point Process $\phi_{p}$ of intensity $\lambda_{P}$, which is called the parent process. For each cluster center $x \epsilon \phi_{p}$, the nodes are distributed according to an independent Poisson Point Process $\phi^{x}$ of intensity $\lambda_{c}$ in the circular covered area of radius $R_{c}$ around the center $x$. The complete distribution of all the node is given as. 


$$
\phi=\bigcup_{x \in \phi_{p}} \phi^{x}
$$

As a channel model we use deterministic distancedependent path loss $R^{-\alpha}$, where $R$ is the distance between the transmitter and the receiver and $\alpha$ is the path loss exponent. We consider Rayleigh fading, $g_{0}$ with $E\left\{g_{0}^{2}\right\}=1$. The interference at the receiver node on the plane is given by

$$
\mathcal{I}_{\phi_{i}}=\sum g_{i}^{2} R_{i}^{-\alpha} \mathcal{P}_{i}
$$

The received SINR $\gamma$ at each device can be expressed as

$$
\gamma=\frac{g_{0}^{2} P_{0} R_{0}^{-\alpha}}{\mathcal{I}_{\phi_{i}}+\sigma_{n}^{2}}
$$

where $P_{0}$ is the transmission power, $R_{0}$ is the distance between the transmitter and the receiver and $\sigma_{n}^{2}$ is the noise power. Then, we can calculate the probabilistic throughput

$$
\operatorname{Pr}\{\gamma>\theta\}=\operatorname{Pr}\left\{\frac{g_{0}^{2} P_{0} R_{0}^{-\alpha}}{\mathcal{I}_{\phi_{i}}+\sigma_{n}^{2}}>\theta\right\}
$$

where $\theta$ is the required SINR for successful reception (threshold). By denoting $m=g^{2}$ this can be deduced to the following form

$$
\begin{aligned}
\operatorname{Pr}\{\gamma>\theta\} & =E\left\{F_{c, m}\left(\frac{\theta R_{0}^{\alpha}\left(\mathcal{I}_{\phi_{i}}+\sigma_{n}^{2}\right)}{P_{0}}\right)\right\} \\
& =E\left\{\exp \left(\frac{-\theta R_{0}^{\alpha}\left(\mathcal{I}_{\phi_{i}}+\sigma_{n}^{2}\right)}{P_{0}}\right)\right\}
\end{aligned}
$$

where $F_{c}($.$) stands for the Complementary Cumulative Distri-$ bution Function (CCDF). Moreover, note that $m$ is an exponential random variable and $F_{c, m}(m)=e^{-m}$. The expectation is taken over the Rayleigh distribution which gives

$$
\begin{aligned}
\operatorname{Pr}\{\gamma>\theta\} & =\int_{0}^{\infty} \operatorname{Pr}\left\{\frac{g_{0}^{2} P_{0} R_{0}^{-\alpha}}{\mathcal{I}_{\phi_{i}}+\sigma_{n}^{2}}>\theta\right\} f_{g}(g) d g \\
& =\int_{0}^{\infty} \operatorname{Pr}\left\{g_{0}^{2}>\frac{\theta R_{0}^{\alpha}\left(\mathcal{I}_{\phi_{i}}+\sigma_{n}^{2}\right)}{P_{0}}\right\} \\
& \times f_{g}(g) d g \\
& =E\left\{\exp \left(\frac{-\mu \theta R_{0}^{\alpha}\left(\mathcal{I}_{\phi_{i}}+\sigma_{n}^{2}\right)}{P_{0}}\right)\right\} \\
& =\exp \left(-\frac{\mu \theta R_{0}^{\alpha} \sigma_{n}^{2}}{P_{0}}\right) \mathcal{L}_{\mathcal{I}_{\phi_{i}}}\left(\frac{\mu \theta R_{0}^{\alpha}}{P_{0}}\right)
\end{aligned}
$$

$\mathcal{L}_{\mathcal{I}_{\phi_{i}}}$ is the laplace transform of interference from CGN and CRN in Poisson cluster process, given by the product of individual laplace transform of CGN and CRN $\left(\mathcal{L}_{\mathcal{I}_{\phi_{i} C G}}\right.$ and $\mathcal{L}_{\mathcal{I}_{\phi_{i C R}}}$ ) respectively, following similar derivation in [12] and [13], we arrived at

$$
\begin{aligned}
\mathcal{L}_{\mathcal{I}_{\phi_{i}}}(s, z) & =\exp \left\{-\lambda_{p} \int_{\mathbb{R}^{2}}[1-\exp (-\bar{c} \vartheta(s, z, y))] d y\right\} \\
& \times \int_{\mathbb{R}^{2}} \exp (-\bar{c} \vartheta(s, z, y)) f(y) d y
\end{aligned}
$$

where

$$
\vartheta(s, z, y)=\int_{\mathbb{R}^{2}} \frac{g(x-y-z)}{s^{-1}+g(x-y-z)} f(x) d x
$$

where $\bar{c}$ is the average number of point in a cluster, $f(x)$ and $f(y)$ are Thomas cluster process, the PDF of the node distribution around its parent point.

\section{B. CRN Per-node Throughput}

In CR networks the received interference depends on the sensing results. Furthermore, in case of overlapping CR networks the operations of the other CR network also affect the performance. Consequently, we have multiple transmission scenarios listed in Table I. In prioritized access, $C G N$ can

Table I: Possible Transmission Probability for $C R N$

\begin{tabular}{lcc}
\hline \hline Scenarios & PU idle $\left(H_{0}^{P U}\right)$ & PU active $\left(H_{1}^{P U}\right)$ \\
\hline CGN idle $\left(H_{0}^{C G}\right)$ & $\left(1-P_{f, 2}^{P U}\right)\left(1-P_{f, 2}^{C G}\right)$ & $P_{m, 2}^{P U}\left(1-P_{f, 2}^{C G}\right)$ \\
\hline CGN active $\left(H_{1}^{C G}\right)$ & $\left(1-P_{f, 2}^{P U}\right) P_{m, 2}^{C G}$ & $P_{m, 2}^{P U} P_{m, 2}^{C G}$ \\
\hline \hline
\end{tabular}

access the medium first if the channel is sensed as idle. $C R N$ is slightly delayed to find out whether $C G N$ started a transmission or not. After that, $C R N$ can transmit if possible. We denote the miss rate and false alarm probabilities of $C G N$ at $C R N$ by $P_{m, 2}^{C G}$ and $P_{f, 2}^{C G}$, and those of $P U$ at $C R N$ by $P_{m, 2}^{P U}$ and $P_{f, 2}^{P U}$, respectively. Similarly, the miss rate and false alarm probabilities of $P U$ at $C G N$ are denoted by $P_{m, 1}^{P U}$ and $P_{f, 1}^{P U}$.

We define multiple capacity scenarios at CRN depending on the situations:

$$
\begin{aligned}
C_{1} & =\operatorname{Pr}\left\{\frac{g_{0}^{2} P_{0} R_{0}^{-\alpha}}{\mathcal{I}_{\phi_{i C R}}+\sigma_{n}^{2}}>\theta\right\} \\
C_{2} & =\operatorname{Pr}\left\{\frac{g_{0}^{2} P_{0} R_{0}^{-\alpha}}{\mathcal{I}_{\phi_{i C R}}+\mathcal{I}_{p}+\sigma_{n}^{2}}>\theta\right\} \\
C_{3} & =\operatorname{Pr}\left\{\frac{g_{0}^{2} P_{0} R_{0}^{-\alpha}}{\mathcal{I}_{\phi_{i C R}}+\mathcal{I}_{\phi_{i C G}}+\sigma_{n}^{2}}>\theta\right\} \\
C_{4} & =\operatorname{Pr}\left\{\frac{g_{0}^{2} P_{0} R_{0}^{-\alpha}}{\mathcal{I}_{\phi_{i C R}}+\mathcal{I}_{\phi_{i C G}}+\mathcal{I}_{p}+\sigma_{n}^{2}}>\theta\right\}
\end{aligned}
$$

For instance, when PU is idle and both CGN and CRN transmit, then the capacity at CRN is given by $C_{3}$. Then the following achievable data rates of $C R N$ can be obtained for different transmission scenarios shown in Table I.

1) Both PU and CGN are idle:

$$
R_{1}=\Omega_{2}\left(1-P_{f, 2}^{P U}\right)\left(1-P_{f, 2}^{C G}\right) C_{1} P\left(H_{0}^{P U}\right) P\left(H_{0}^{C G}\right)
$$

where $\Omega_{2}=\left(T-\tau_{2}\right) / T$.

2) $\mathrm{PU}$ is active but $C G N$ remains idle:

$$
R_{2}=\Omega_{2} P_{m, 2}^{P U}\left(1-P_{f, 2}^{C G}\right) C_{2} P\left(H_{1}^{P U}\right) P\left(H_{0}^{C G}\right)
$$


3) $\mathrm{PU}$ is idle, but $C G N$ is active:

$$
R_{3}=\Omega_{2}\left(1-P_{f, 2}^{P U}\right) P_{m, 2}^{C G} C_{3} P\left(H_{0}^{P U}\right) P\left(H_{1}^{C G}\right)
$$

4) Both PU and CGN are active:

$$
R_{4}=\Omega_{2} P_{m, 2}^{P U} P_{m, 2}^{C G} C_{4} P\left(H_{1}^{P U}\right) P\left(H_{1}^{1}\right)
$$

We define the per-node throughput such that the transmitter has a packet to transmit while a receiver is idle, i.e., the receiver does not have a packet to transmit. This can be formulated as follows

$$
H=p(1-p) R_{T}
$$

where $R_{T}=R_{1}+R_{2}+R_{3}+R_{4}$ and $p$ is the transmission probability.

\section{Performance Bound on Spectrum Sensing of CRN}

In practice $\mathrm{CR}$ users should achieve reasonable data rate, we denote this rate as $\hat{H}$. Next, we derived the bound of the false alarm probability that is required to achieve the desired rate $H \geq \hat{H}$.

By analyzing $R_{1}, R_{2}, R_{3}$, and $R_{4}$, we concluded that in practice $R_{2}$ and $R_{4}$ have negligible influence on the performance of CR users since both the miss rate probability and the probability of PU being active are small. Thus, the following approximation is applied.

$$
\begin{aligned}
H & \geq \hat{H} \Longrightarrow \\
\hat{H} & \leq p(1-p) \Omega_{2}\left(1-P_{f, 2}^{P U}\right)\left(1-P_{f, 2}^{C G}\right) C_{1} P\left(H_{0}^{P U}\right) P\left(H_{0}^{C G}\right) \\
& +p(1-p) \Omega_{2}\left(1-P_{f, 2}^{P U}\right) P_{m, 2}^{C G} C_{3} P\left(H_{0}^{P U}\right) P\left(H_{1}^{C G}\right)
\end{aligned}
$$

Define

$$
\begin{aligned}
& \eta_{1}=\Omega_{2} C_{1} P\left(H_{0}^{P U}\right) P\left(H_{0}^{C G}\right) \\
& \eta_{2}=\Omega_{2} C_{3} P\left(H_{0}^{P U}\right) P\left(H_{1}^{C G}\right)
\end{aligned}
$$

and assume that CRN has the same spectrum sensing performance for CGN and PU, i.e., $P_{f, 2}^{P U}=P_{f, 2}^{C G}=P_{f, 2}$ and $P_{m, 2}^{P U}=P_{m, 2}^{C G}=P_{m, 2}$. Then,

$$
\hat{H} \leq p(1-p)\left(1-P_{f, 2}\right)\left[\left(1-P_{f, 2}\right) \eta_{1}+P_{m, 2} \eta_{2}\right]
$$

If $P_{f, 2}$ and $P_{m, 2}$ are very small, the achievable rate for a node approaches $p(1-p) \eta_{1}$. It can also be shown that the achievable rate of a node will decrease when $P_{f, 2}$ increases and $P_{m, 2}$ decreases.

If the spectrum sensing performance of CGN at CRN is given a priori, then we can find the maximum probability of false alarm of PU at CRN for achieving a certain rate $\hat{H}$

$$
P_{f, 2}^{P U} \leq 1-\frac{\hat{H}}{\left[\left(1-P_{f, 2}^{C G}\right) \eta_{1}+P_{m, 2}^{C G} \eta_{2}\right] p(1-p)}
$$

The above equation (22) defines the upper bound for probability of false alarm of PU at CRN.

\section{CGN Performance}

It is relatively straightforward to calculate the achievable rates of $C G N$ assuming the miss rate probability of CGN at CRN is very low. From Table II and the capacity formulas given below

$$
\begin{aligned}
& C_{1}^{C G}=\operatorname{Pr}\left\{\frac{g_{0}^{2} P_{0} R_{0}^{-\alpha}}{\mathcal{I}_{\phi_{i C G}}+\sigma_{n}^{2}}>\theta\right\} \\
& C_{2}^{C G}=\operatorname{Pr}\left\{\frac{g_{0}^{2} P_{0} R_{0}^{-\alpha}}{\mathcal{I}_{\phi_{i C G}}+\mathcal{I}_{p}+\sigma_{n}^{2}}>\theta\right\}
\end{aligned}
$$

Table II: Possible Transmission Probabilities for $C G N$

\begin{tabular}{ccc}
\hline \hline Scenarios & PU idle $\left(H_{0}^{P U}\right)$ & PU active $\left(H_{1}^{P U}\right)$ \\
\hline & $\left(1-P_{f, 1}^{P U}\right)$ & $P_{m, 1}^{P U}$ \\
\hline \hline
\end{tabular}

The achievable rates are

1) $\mathrm{PU}$ is idle:

$$
R_{1}^{C G}=\Omega_{1}\left(1-P_{f, 1}^{P U}\right) C_{1}^{C G} P\left(H_{0}^{P U}\right)
$$

where $\Omega_{1}=\left(T-\tau_{1}\right) / T$.

2) $\mathrm{PU}$ is active:

$$
R_{2}^{C G}=\Omega_{1} P_{m, 1}^{P U} C_{2}^{C G} P\left(H_{1}^{P U}\right)
$$

Similarly, the bound on the spectrum sensing of PU at $C G N$ should satisfy

$$
P_{f, 1}^{P U} \leq 1-\frac{\hat{H}_{1}}{\left[\Omega_{1} C_{1}^{C G} P\left(H_{0}^{P U}\right)\right] p_{1}\left(1-p_{1}\right)}
$$

where $\hat{H}_{1}$ is the desired data rate of CG and $p_{1}$ is the transmission probability of CG.

\section{Results AND ANALYsis}

Based on the analysis and derivations presented above, the achievable rate of the prioritized access is presented in this section. The performance of overlapping CR networks is studied by investigating the effects of different parameters on the achievable rate of CRN under prioritized access. In this section, we use the following practical values for network parameters: $R_{0}=1, P_{0}=30 \mathrm{dBm}, \sigma_{n}=-70 \mathrm{dBm}, \theta=10$ $\mathrm{dB}, \bar{c}=3, \alpha=4, p_{1}=p_{2}=0.5, \lambda_{p}=2$, and $\mu=1$. For each resulted figure we vary different parameters to demonstrate their impact.

Figure 3 and Figure 4 show the effect of sensing performance (false alarm probability and miss rate probability) on the achievable rate in case of overlapping CR networks under prioritize secondary access. These figures capture the fundamental nature of overlapping CR networks. As false alarm probability of CR network increase, the achievable rate decreases. Miss rate probability has the opposite effect, because increase in miss rate probability increases the achievable rate. These results imply that CR users would like to have as low probability of false alarm as possible while keeping the 


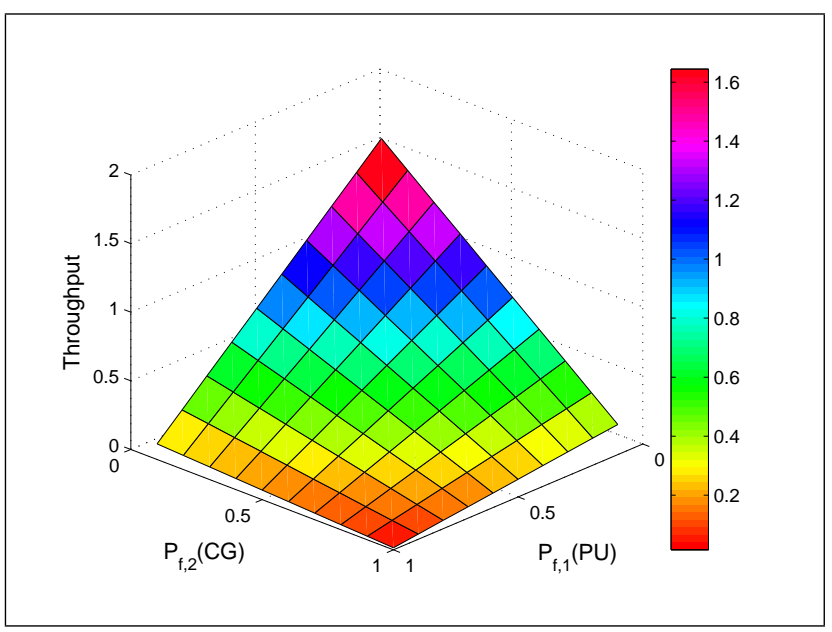

Figure 3: Effect of false alarm probability on the Throughput of CRN

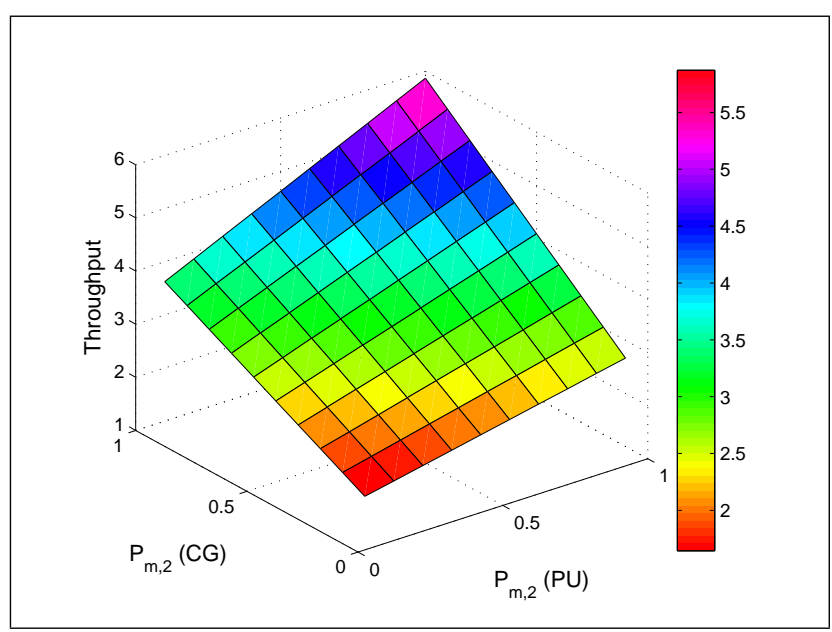

Figure 4: Effect of miss rate probability on the Throughput of CRN

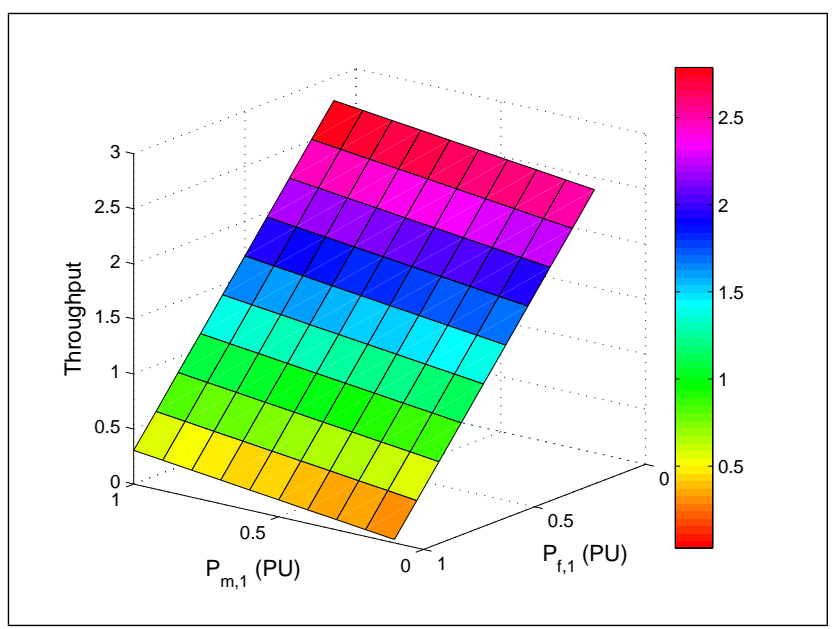

Figure 5: Effect of false alarm and miss probabilities on the Throughput of CGN required miss rate probability (due to regulations) to achieve the best performance. The false alarm probability of CGN network should be high such that the CRN network would be able to access and use the spectrum alone as often as possible.

Figure 5 shows the achievable rate for CGN. It demonstrates similar effect of spectrum sensing performance on the achievable rate, i.e., higher false alarm probability implies less data rate and higher miss rate probability implies more data rates.

\section{RElATED WORKS AND CONTRIBUTION}

There are many works on the coexistence of heterogeneous wireless networks in the ISM bands, such as the coexistence of WiFi (802.11) and Zigbee (802.15.4) radios [1]-[3], [8], [14], [15]. IEEE $802.11 \mathrm{~b} / \mathrm{g}$ networks may interfere with IEEE 802.15.4 sensor networks and thereby introduce significant coexistence problems for low-power sensor nodes [1], [2]. In [14], a coexistence model of IEEE 802.15.4 and IEEE $802.11 \mathrm{~b} / \mathrm{g}$, which exposes the interactive behavior between these two standards and therefore accurately explains their coexistence performance was proposed. The authors of [8] used a multi-agent system based approach to achieve information sharing and decision distribution among multiple 802.11 networks deployed within small geographic vicinity. An experimental study was performed in [15], where the results raise important coexistence issues for 802.15.4 and 802.11 by showing that 802.15 .4 significantly impacts 802.11 performance in many cases. The more recent study [3] proposed a novel MAC, Cooperative Busy Tone (CBT), that enables the reliable coexistence between WiFi and Zigbee. CBT allows a separate ZigBee node to schedule a busy tone concurrently with the desired transmission, thereby improving the visibility of ZigBee devices to WiFi. However, these works focus on the coexistence of heterogeneous wireless networks in the ISM bands, rather than the coexistence of multiple homogeneous cognitive radio networks with primary user network. Furthermore, the analysis were mainly on the different PHY/MAC structures and standards of WiFi (802.11) and Zigbee (802.15.4) radios.

Although intensive research has been carried out on $\mathrm{CR}$ technology and single CR networks, only a few studies address the coexistence of multiple CR networks [5]-[7], [9], [10]. In [5], customer admission and eviction control was investigated using game theory for two co-located wireless service providers that temporarily lease a licensed spectrum band from the licensees and opportunistically utilize it during the absence of the legacy users. The goal is to provide WiFilike Internet access in spectrum whitespaces with better service quality than that of WiFi in the ISM band. The minimum blocking probabilities and maximum spectrum utilizations of three co-located systems with different bandwidth requirements were derived for one-channel band scenario in [6]. A channel packing scheme was then proposed for the multiplechannel band scenario to decrease the blocking probability and reduce the overall failure probability of the cognitive radio systems. A priority queue model was proposed for cognitive radio networks in [7], where the primary user has preemptive 
priority while the cognitive users are further divided into different priority levels. A scheduling model was built based on the hybrid priority dynamic policy. In [9], a system model for dynamic spectrum access consisting of two CR networks with intra-group cooperation and inter-group competition was developed, the intra-group cooperation was implemented using opportunistic decode-and-forward (ODF) scheme to demonstrate the effects of intra-group cooperation in an underlay spectrum sharing network and its impact on enhancing group performance. In [10], three state sensing model was proposed to detect the PU active and idle state as well as the SU activities in multiple CR networks. It is shown that the scheduler provided much needed gain during congestions. However, none of the above works discussed the fundamental per-node throughput of a cognitive radio user when multiple cognitive radio networks coexist with primary users. The authors in [4] discussed the fundamental per-node throughput of a CR user when multiple CR networks coexist under simultaneous access with the PU but did not consider the performance in prioritized overlapping CR networks. Furthermore, we provide insights on the dominant factors of the per-node throughput and validated these in the results.

\section{CONCLUSIONS}

In this paper, we investigated the performance of two coexisting cognitive radio networks with a primary network. Specifically, we consider the case where one cognitive radio network, a cognitive gateway network (CGN), has higher priority to access spectrum than the other cognitive radio network (CRN). The achievable rates in both CGN and CRN are derived and the performance bounds in terms of false alarm probability are given for desired data rate. These results can be used in the planning and deployment of multiple cognitive radio networks with different priorities.

\section{ACKNOWLEDGMENT}

This research work is supported in part by the US NSF under CNS-1040207 and the US Army Research Office under W911NF-12-1-0054 and W911NF-14-1-0044.

\section{REFERENCES}

[1] S. Shin, H. Park, and W. Kwon, "Mutual Interference Analysis of IEEE 802.15.4 and IEEE 802.11b," Computer Networks, vol. 51, no. 12, pp. 3338-3353, August 2007.
[2] J. Huang, G. Xing, G. Zhou, and R. Zhou, "Beyond Co-Existence: Exploiting WiFi White Space for Zigbee Performance Assurance," in Proceedings of the 18th IEEE International Conference on Network Protocols, Kyoto, Japan, October 2010, pp. 305-314.

[3] X. Zhang and K. G. Shin, "Enabling Coexistence of Heterogeneous Wireless Systems: Case for ZigBee and WiFi," in Proceedings of the 12th ACM International Symposium on Mobile Ad Hoc Networking and Computing, Paris, France, May 2011, pp. 1-11.

[4] J. Nieminen, L. Qian, and R. Jantti, "Per-node throughput performance of overlapping cognitive radio networks," in Cognitive Radio Oriented Wireless Networks and Communications (CROWNCOM), 2012 7th International ICST Conference on, 2012, pp. 163-168.

[5] H. Kim, J. Choi, and K. Shin, "Wi-Fi 2.0: Price and Quality Competitions of Duopoly Cognitive Radio Wireless Service Providers with Time-Varying Spectrum Availability," in Proceedings of the 30th Annual IEEE International Conference on Computer Communications, Shanghai, China, April 2011, pp. 2453-2461.

[6] L. Luo and S. Roy, "Analysis of Dynamic Spectrum Access with Heterogeneous Networks: Benefits of Channel Packing Scheme," in Proceedings of the IEEE Global Telecommunications Conference, Honolulu, HI, USA, December 2009, pp. 1-7.

[7] P. Zhu, J. Li, and X. Wang, "Scheduling Model for Cognitive Radio," in Proceedings of the 3rd International Conference on Cognitive Radio Oriented Wireless Networks and Communications, Singapore, Singapore, May 2008, pp. 1-6.

[8] J. Xie, I. Howitt, and A. Raja, "Cognitive Radio Resource Management Using Multi-Agent Systems," in Proceedings of the 4th IEEE Consumer Communications and Networking Conference, Las Vegas, NV, USA, January 2007, pp. 1123-1127.

[9] S. Chen, C. Ghosh, A. M. Wyglinski, and S. Jayaweera, "Impact of group cooperation over competitive secondary subnetworks," in Communications, Computers and Signal Processing (PacRim), 2011 IEEE Pacific Rim Conference on, 2011, pp. 768-773.

[10] Y. Zhao, M. Song, C. Xin, and M. Wadhwa, "Spectrum sensing based on three-state model to accomplish all-level fairness for co-existing multiple cognitive radio networks," in INFOCOM, 2012 Proceedings IEEE, 2012, pp. $1782-1790$

[11] IEEE Std 802.22.1-2010, "IEEE Standard for Information Technology - Telecommunications and Information Exchange between Systems Local and Metropolitan Area Networks - Specific Requirements - Part 22.1: Standard to Enhance Harmful Interference Protection for LowPower Licensed Devices Operating in TV Broadcast Bands," November 2010.

[12] R. Ganti and M. Haenggi, "Interference and outage in clustered wireless ad hoc networks," Information Theory, IEEE Transactions on, vol. 55, no. 9, pp. 4067-4086, 2009.

[13] C. han Lee and M. Haenggi, "Interference and outage in poisson cognitive networks," Wireless Communications, IEEE Transactions on, vol. 11, no. 4, pp. 1392-1401, 2012.

[14] W. Yuan, X. Wang, and J.-P. Linnartz, "A coexistence model of ieee 802.15 .4 and ieee $802.11 \mathrm{~b} / \mathrm{g}$," in 14th IEEE Symposium on Communications and Vehicular Technology in the Benelux, nov. 2007, pp. 1 -5.

[15] S. Pollin, I. Tan, B. Hodge, C. Chun, and A. Bahai, "Harmful coexistence between 802.15.4 and 802.11: A measurement-based study," in 3rd International Conference on Cognitive Radio Oriented Wireless Networks and Communications (CrownCom), may 2008, pp. 1-6. 